\title{
Machine Directional Register System Modeling for Shaft-Less Drive Gravure Printing Machines
}

\author{
Shanhui Liu, ${ }^{1}$ Xuesong Mei, ${ }^{1}$ Jian Li, ${ }^{2}$ and Li'e $\mathrm{Ma}^{1}$ \\ ${ }^{1}$ School of Mechanical Engineering, Xian Jiaotong University, Xianning West Road 28, Xian, Shaanxi 710049, China \\ ${ }^{2}$ School of Mechatronics Engineering, Henan University of Science and Technology, Xiyuan Road 48, Luoyang, Henan 471003, China \\ Correspondence should be addressed to Shanhui Liu; shanhuiliu820404@163.com
}

Received 22 June 2013; Accepted 8 September 2013

Academic Editor: William Guo

Copyright (c) 2013 Shanhui Liu et al. This is an open access article distributed under the Creative Commons Attribution License, which permits unrestricted use, distribution, and reproduction in any medium, provided the original work is properly cited.

\begin{abstract}
In the latest type of gravure printing machines referred to as the shaft-less drive system, each gravure printing roller is driven by an individual servo motor, and all motors are electrically synchronized. The register error is regulated by a speed difference between the adjacent printing rollers. In order to improve the control accuracy of register system, an accurate mathematical model of the register system should be investigated for the latest machines. Therefore, the mathematical model of the machine directional register (MDR) system is studied for the multicolor gravure printing machines in this paper. According to the definition of the MDR error, the model is derived, and then it is validated by the numerical simulation and experiments carried out in the experimental setup of the four-color gravure printing machines. The results show that the established MDR system model is accurate and reliable.
\end{abstract}

\section{Introduction}

Recently, with the development of high-speed and highprecision printing, shaft-less drive technology has been widely used in the latest gravure printing machines. In this technology, the printing rollers are driven by independent servo motors, and the register error is controlled through directly adjusting the speed of the pertinent servo motor instead of changing the position of the compensation roller, so the traditional mechanical shaft is eliminated to make printing faster and more accurate. This register mode has the advantages such as higher response frequency, better control accuracy, and lower additional cost. However, the register error in the shaft-less drive mode has a cumulative effect; namely, the adjustment of register error in upstream will affect all register errors in downstream. Hence, the mathematical model of the register system which can accurately reflect the variation of the register errors is needed to be studied to control the register errors accurately.

It is difficult to get an exact register system model because the register errors are affected by various factors, such as the web tension and speed, the precision of parts and assembling, and the web material and production environment. Register errors are defined as two-dimensional errors: machine directional register (MDR) errors and cross-directional register (CDR) errors. Brandenburg [1] derived a linear mathematical model of the MDR errors of a moving web in a first-order differential equation by using an equilibrium equation of mass which is transported by printing rollers, but this model was based on the mechanical shaft drive mode and could not adapt to the shaft-less drive mode. Yoshida et al. [24] developed the MDR system model for the mechanical shaft and shaft-less drive mode, but the model still ignores many influencing factors and is imperfect. Hua et al. [5] got the MDR errors model of gravure printing machines via the system identification method, but this method has poor universality and bad practicability. Although the MDR errors model of the two-color system was established in [6], the MDR system model of the multicolor system was not derived, some key deducing steps were ignored, and some wrong symbols appeared in some formulae, which makes some processes of derivation difficult to be understood for readers. The modeling and control of CDR system were studied in [79]. The CDR errors are controlled by the lateral motion of printing rollers, which is greatly different from the control of MDR errors. In addition, initial works of modeling and 


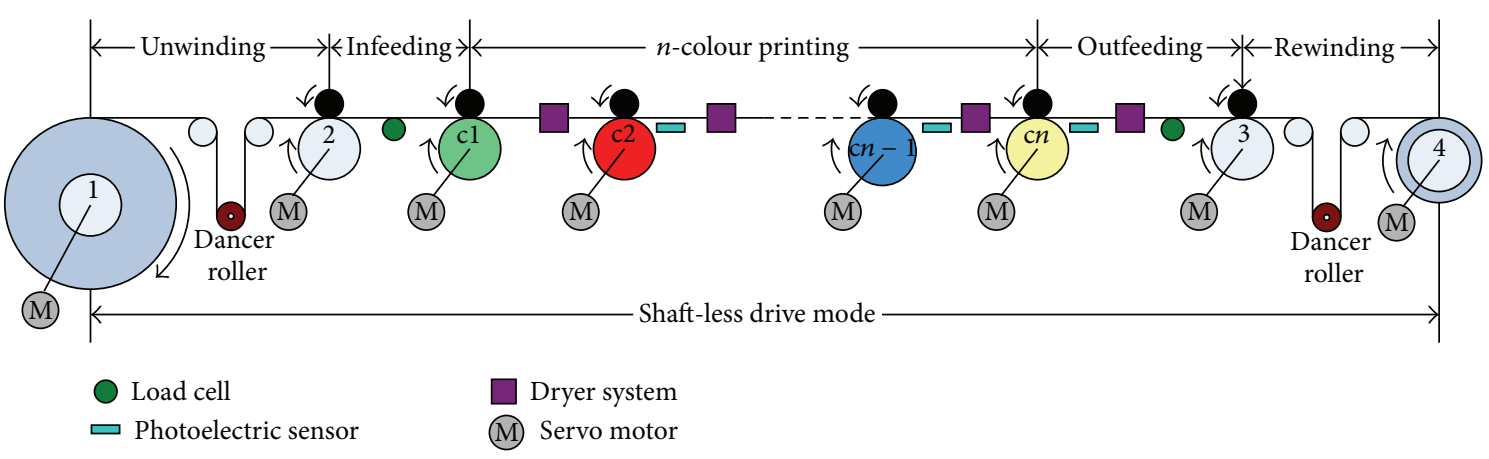

FIGURE 1: Schematic diagram of the $n$-color gravure printing machines.

control in correlation between MDR and CDR errors were researched in $[10,11]$. However, the in-depth research on the MDR system model based on shaft-less drive mode is still not enough in gravure printing machines.

Therefore, in this study, a mathematical model of the MDR system in shaft-less drive mode is derived for gravure printing machines by using an equilibrium equation of mass and considering the fluctuation of tension, speed, and other factors. This paper is structured as follows. Section 2 introduces the schematic diagram of shaft-less drive gravure printing machines. Section 3 establishes the MDR errors model of the two-color system and then obtains the MDR system model of the multicolor gravure printing machines. Section 4 verifies the derived model by simulation and experiments. The results show that the model is accurate enough to describe the MDR errors in shaft-less drive gravure printing machines.

\section{Shaft-Less Drive Gravure Printing Machines}

The schematic diagram of the $n$-color gravure printing machines is presented in Figure 1, and $n$ stands for the total number of printing units. This system is composed of an unwinding unit, an infeeding unit, an $n$-color printing system, an outfeeding unit, and a rewinding unit. Shaftless drive mode has been used in the machines; that is, all of the driving shafts are driven by independent servo motors. The $n$-color printing system consists primarily of $n$ printing rollers, and $\mathrm{cl}$ is the first printing roller whose main task is to maintain the system speed constant. In the printing subsystem, tension is same with that in infeeding zone at steady state. Load cells are installed at idle rollers in the middle of continuous process in both the infeeding and outfeeding units for tension pickup. Two passive dancer rollers are used to reduce tension fluctuations and measure the tension signals simultaneously in the unwinding and rewinding units. Photoelectric sensors are installed in the machine for registering errors pickup.

Control of the gravure printing machines is basically composed of tension control and register control. Register control is required for high printing resolution in the multistage printing systems. Hence, an accurate MDR system model is essential to ensure adequate printing performance for the gravure printing machines.

\section{Mathematical Modeling}

3.1. Basic Assumption. In the gravure printing machines, the MDR error of a moving web is defined in two adjacent printing rollers as the relative difference of the distance between the previous printed pattern by the upstream printing roller and the later printed one in the downstream printing roller. To remove unnecessary interference and simplify the mathematical model, the following assumptions are made in the derivation of model.

(1) There is no slippage between web material and rollers and no mass transfer between the web material and the environment.

(2) The web material is isotropic, and its properties do not change with temperature and humidity.

(3) The web cross-section in the unstretched state does not vary along machine direction in a web span.

(4) The density and modulus of elasticity of the web in the unstretched state are constant in the cross-section.

(5) The strain in web is small (much less than unity) and uniform within the web span.

(6) The printing system is in a stable state, in which there are no disturbances from tension and other factors in time $t \leq 0$.

3.2. MDR Error Model of the Two-Color System. The mathematical MDR error model of the two-color system in shaftless drive gravure printing machines is illustrated in Figure 2 by the coupling between the $i$ th and $(i+1)$ th units, and the $i$ th unit is register errors reference. As shown in Figure 2, a coordinate axis along the machine direction is set up and the position of the $i$ th unit is set as the origin of the coordinate axis. Figures 2(b) and 2(c) show that the color marks on printing roller $c i$ and $c i+1$ are printed on the web at the printing instants $t_{1}$ and $t_{2}$, respectively.

The MDR error in the moving coordinate system is to calculate the difference between the printing point on the roller and the printed point on the web representing 


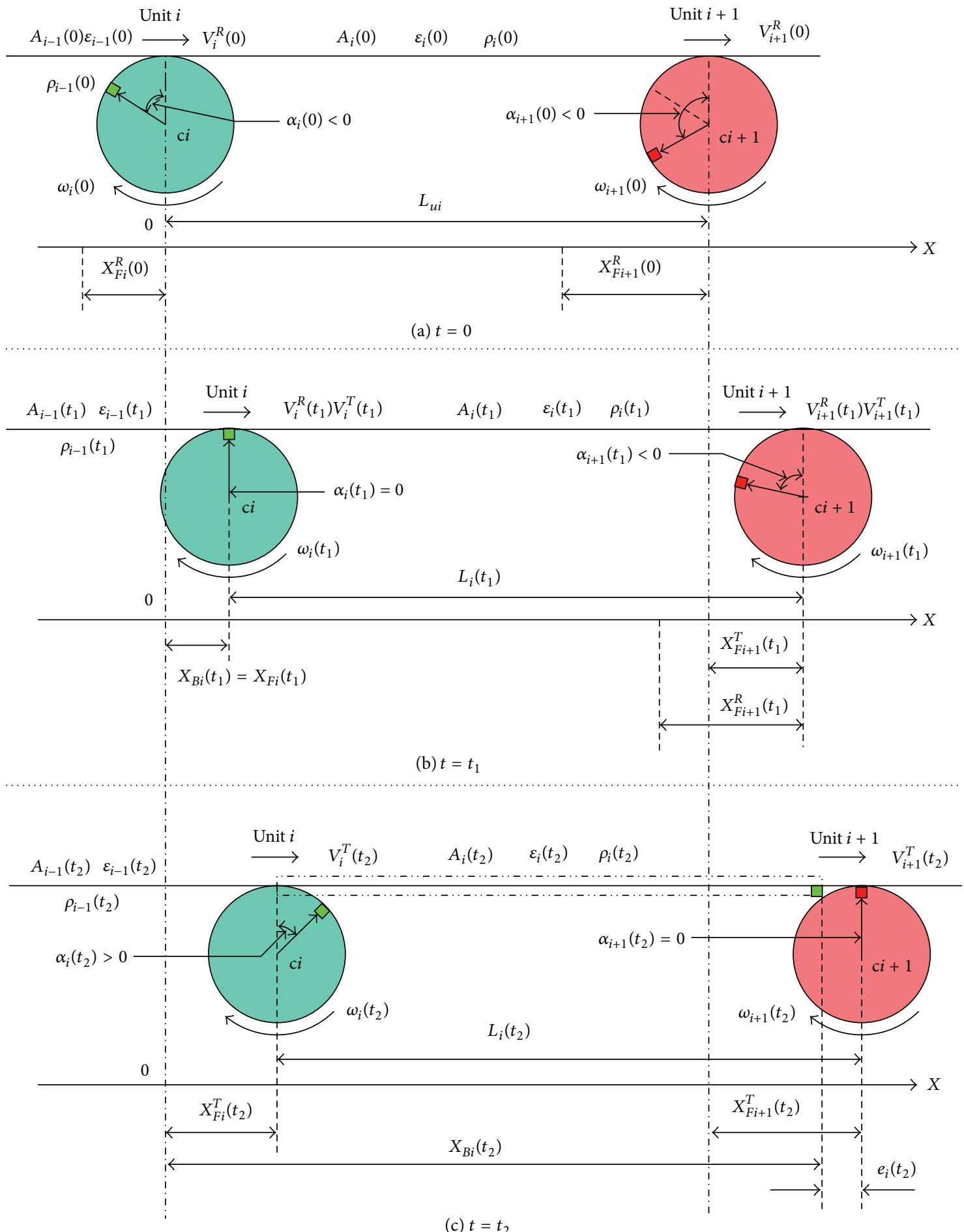

FIGURE 2: MDR error model of the two-color system.

the same point of the pattern at the printing instants. Therefore, according to the moving coordinate system, the MDR error is just the deviation from the coordinate of color mark on the printing roller $X_{F i+1}$ and that on the web $X_{B i}$. At instant $t_{2}$, the MDR error is defined by

$$
e_{i}\left(t_{2}\right)=X_{B i}\left(t_{2}\right)-X_{F i+1}\left(t_{2}\right) \text {. }
$$

It is to be noted that the movement of the printing roller can be viewed as a combination of two movements: one is the rotation of the roller and the other is the virtual translational movement of the roller along the machine direction which is caused by the variation of the web length. This variation can be affected by many factors such as dryer system, tension sensors, or some other disturbances. 
By using assumptions (1) and (2), the following conclusion can be obtained:

$$
\begin{aligned}
& \frac{d}{d t} \int_{0}^{L_{i}} \rho_{i}(x, t) A_{i}(x, t) d x \\
& \quad=\rho_{i-1}(x, t) A_{i-1}(x, t) V_{i}^{R}(t)-\rho_{i}(x, t) A_{i}(x, t) V_{i+1}^{R}(t) .
\end{aligned}
$$

Hence, the mass of the web material entering the printing unit $i$ in the time interval from $t_{1}$ to $t_{2}$ can be derived as follows:

$$
\frac{d}{d t} \int_{X_{B i}\left(t_{1}\right)}^{X_{B i}\left(t_{2}\right)} \rho_{i}(x, t) A_{i}(x, t) d x=\rho_{i-1}(x, t) A_{i-1}(x, t) V_{i}^{R}(t) .
$$

According to assumptions (3) and (4) and the definition of strain, the relationship between the stretched and unstretched webs can be written as follows:

$$
\frac{\rho_{i}(x, t) A_{i}(x, t)}{\rho_{u} A_{u}}=\frac{1}{1+\varepsilon_{i}(x, t)},
$$

where $u$ means the unstretched state of the web.

Combining (3) with (4), we can obtain

$$
\int_{X_{B i}\left(t_{1}\right)}^{X_{B i}\left(t_{2}\right)} \frac{1}{1+\varepsilon_{i}(x, t)} d x=\int_{t_{1}}^{t_{2}} \frac{V_{i}^{R}(t)}{1+\varepsilon_{i-1}(x, t)} d t .
$$

Under assumption (5), we can obtain

$$
\frac{1}{1+\varepsilon_{i}(x, t)}=\frac{1}{1+\varepsilon_{i}(t)} \approx 1-\varepsilon_{i}(t) .
$$

Combining (5) with (6), we can get

$$
\left(X_{B i}\left(t_{2}\right)-X_{B i}\left(t_{1}\right)\right)\left(1-\varepsilon_{i}(t)\right)=\int_{t_{1}}^{t_{2}}\left(1-\varepsilon_{i-1}(t)\right) V_{i}^{R}(t) d t .
$$

As shown in Figure 2(a), the initial phase angle of the color mark on the printing roller $c i$ is negative and can be calculated by

$$
\alpha_{i}(t)=\alpha_{i}(0)+\int_{0}^{t} \omega_{i}(t) d t .
$$

The coordinate of color mark on the printing roller $\mathrm{ci} X_{F i}$ can be calculated by (9) including two parts, $X_{F i}^{R}$ and $X_{F i}^{T}$, which are caused by rotation and virtual translational movement of the printing roller ci, respectively:

$$
\begin{aligned}
X_{F i}(t) & =X_{F i}^{R}(t)+X_{F i}^{T}(t) \\
& =X_{F i}^{R}(0)+X_{F i}^{T}(0)+\int_{0}^{t}\left[V_{i}^{R}(t)+V_{i}^{T}(t)\right] d t .
\end{aligned}
$$

Under assumption (6), the following equation could be obtained

$$
\begin{gathered}
X_{F i}^{R}(0)=R_{i} \alpha_{i}(0) \leq 0, \\
X_{F i}^{T}(0)=0 .
\end{gathered}
$$

So, (9) can be rewritten as follows:

$$
X_{F i}(t)=R_{i} \alpha_{i}(0)+\int_{0}^{t}\left[V_{i}^{R}(t)+V_{i}^{T}(t)\right] d t .
$$

At $t=t_{1}$, as shown in Figure 2(b), the color mark on the printing roller $\mathrm{c} i$ is printed on the web, the following equation can be gotten

$$
\alpha_{i}\left(t_{1}\right)=\alpha_{i}(0)+\int_{0}^{t_{1}} \omega_{i}(t) d t=0 .
$$

We can rewrite (12) as follows:

$$
\int_{0}^{t_{1}} \omega_{i}(t) d t=-\alpha_{i}(0) \Longrightarrow \int_{0}^{t_{1}} V_{i}^{R}(t) d t=-R_{i} \alpha_{i}(0) .
$$

The coordinate of the color mark on the printing roller $c i$ coincides with that of the color mark on the web at $t=t_{1}$, so combining (11) with (13), we can get the following:

$$
X_{F i}\left(t_{1}\right)=X_{B i}\left(t_{1}\right)=\int_{0}^{t_{1}} V_{i}^{T}(t) d t .
$$

Because the $i$ th unit is MDR error reference, the printing roller c $i$ has no virtual translational movement, so $V_{i}^{T}(t)=0$, and we can obtain

$$
X_{B i}\left(t_{1}\right)=\int_{0}^{t_{1}} V_{i}^{T}(t) d t=0 .
$$

Hence, (7) can be rewritten as follows:

$$
X_{B i}\left(t_{2}\right)=\frac{1}{1-\varepsilon_{i}(t)} \int_{t_{1}}^{t_{2}}\left(1-\varepsilon_{i-1}(t)\right) V_{i}^{R}(t) d t .
$$

Similarly, as shown in Figure 2, the coordinate of the color mark on the printing roller ci+1 can be derived by.

$$
\begin{aligned}
X_{F i+1}(t) & =X_{F i+1}^{R}(t)+X_{F i+1}^{T}(t) \\
& =X_{F i+1}^{R}(0)+X_{F i+1}^{T}(0)+\int_{0}^{t}\left[V_{i+1}^{R}(t)+V_{i+1}^{T}(t) d t\right] .
\end{aligned}
$$

At $t=0$, (18) can be obtained as follows:

$$
X_{F i+1}^{T}(0)=L_{u i} \text {. }
$$

Derived with the same procedure as $X_{F i}$, the following conclusion can be obtained:

$$
\int_{0}^{t_{2}} V_{i+1}^{R}(t) d t=-X_{F i+1}^{R}(0) .
$$

Combining (17), (18) with (19), we can get

$$
X_{F i+1}\left(t_{2}\right)=X_{B i+1}\left(t_{2}\right)=L_{u i}+\int_{0}^{t_{2}} V_{i+1}^{T}(t) d t .
$$

From the literature [12], we can obtain

$$
\begin{aligned}
& {\left[1-\varepsilon_{i}(t)\right] \frac{d L_{i}(t)}{d t}-L_{i}(t) \frac{d \varepsilon_{i}(t)}{d t}} \\
& \quad=V_{i}^{R}(t)-V_{i+1}^{R}(t)-\varepsilon_{i-1}(t) V_{i}^{R}(t)+\varepsilon_{i}(t) V_{i+1}^{R}(t) .
\end{aligned}
$$


Since $d \varepsilon_{i}(t) / d t \approx 0,(21)$ can be rewritten as follows:

$$
\frac{d L_{i}(t)}{d t}=\frac{1-\varepsilon_{i-1}(t)}{1-\varepsilon_{i}(t)} V_{i}^{R}(t)-V_{i+1}^{R}(t)
$$

Because the change of the web length mapped to the coordinate is equivalent to the speed difference between the virtual translational movements of the two printing rollers, it can be expressed as follows:

$$
\frac{d L_{i}(t)}{d t}=V_{i+1}^{T}(t)-V_{i}^{T}(t)
$$

Due to $V_{i}^{T}(t)=0$, combining (22) with (23), (24) can be obtained as follows:

$$
V_{i+1}^{T}(t)=\frac{1-\varepsilon_{i-1}(t)}{1-\varepsilon_{i}(t)} V_{i}^{R}(t)-V_{i+1}^{R}(t) .
$$

Combining (20) with (24), (20) can be rewritten as follows:

$$
X_{F i+1}\left(t_{2}\right)=L_{u i}+\int_{0}^{t_{2}}\left[\frac{1-\varepsilon_{i-1}(t)}{1-\varepsilon_{i}(t)} V_{i}^{R}(t)-V_{i+1}^{R}(t)\right] d t .
$$

By using assumptions (5), we can get the following:

$$
\begin{gathered}
\frac{1}{1-\varepsilon_{i}(t)} \approx 1+\varepsilon_{i}(t), \\
\varepsilon_{i-1}(t) \varepsilon_{i}(t) \approx 0 .
\end{gathered}
$$

Therefore, (25) can be simplified as follows:

$$
\begin{aligned}
X_{F i+1}\left(t_{2}\right)=L_{u i}+\int_{0}^{t_{2}} & {\left[V_{i}^{R}(t)-V_{i+1}^{R}(t)\right.} \\
& \left.-\left(\varepsilon_{i-1}(t)-\varepsilon_{i}(t)\right) V_{i}^{R}(t)\right] d t
\end{aligned}
$$

According to the definition of MRD error, and combining (1), (16), and (27), the MDR error between the $i$ th and unit $(i+1)$ th printing units at the instant $t_{2}$ can be calculated as follows:

$$
\begin{aligned}
e_{i}\left(t_{2}\right)= & \frac{1}{1-\varepsilon_{i}(t)} \int_{t_{1}}^{t_{2}}\left(1-\varepsilon_{i-1}(t)\right) V_{i}^{R}(t) d t-L_{u i} \\
& -\int_{0}^{t_{2}}\left[V_{i}^{R}(t)-V_{i+1}^{R}(t)-\left(\varepsilon_{i-1}(t)-\varepsilon_{i}(t)\right) V_{i}^{R}(t)\right] d t .
\end{aligned}
$$

The running time $t_{T i}=t_{2}-t_{1}$ represents the time that a color mark on the web takes to move from printing roller $c i$ to printing roller $c i+1$, so $t_{1}=t_{2}-t_{T i},(28)$ can be simplified as follows:

$$
\begin{aligned}
e_{i}\left(t_{2}\right)= & \int_{0}^{t_{2}}\left(V_{i+1}^{R}(t)-\varepsilon_{i}(t) V_{i}^{R}(t)\right) d t \\
& -\int_{0}^{t_{2}-t_{T i}}\left(1-\varepsilon_{i-1}(t)\right) V_{i}^{R}(t) d t-L_{u i} .
\end{aligned}
$$

Extending (28) to any time interval, then we can get

$$
\begin{aligned}
e_{i}(t)= & \int_{0}^{t}\left[V_{i+1}^{R}(t)-\varepsilon_{i}(t) V_{i}^{R}(t)\right] d t \\
& -\int_{0}^{t-t_{T i}}\left(1-\varepsilon_{i-1}(t)\right) V_{i}^{R}(t) d t-L_{u i} .
\end{aligned}
$$

The differential form of MDR error between the $i$ th and $(i+1)$ th units can be derived as follows:

$$
\begin{aligned}
\frac{d e_{i}(t)}{d t}= & V_{i+1}^{R}(t)-V_{i}^{R}\left(t-t_{T i}\right) \\
& +\varepsilon_{i-1}\left(t-t_{T i}\right) V_{i}^{R}\left(t-t_{T i}\right)-\varepsilon_{i}(t) V_{i}^{R}(t) .
\end{aligned}
$$

In the shaft-less drive printing system, there is no change of the web length caused by compensation rollers, so $\Delta L_{i}(t)$ is mainly caused by load cells, dryer systems, and other disturbances. In practice, $\Delta L_{i}(t) \ll L_{u i}$, so we can obtain

$$
L_{i}(t)=L_{u i}+\Delta L_{i}(t) \approx L_{u i}
$$

In (21), we substitute $L_{u i}$ for $L_{i}(t)$, and then (21) can be rewritten as follows:

$$
L_{u i} \frac{d \varepsilon_{i}(t)}{d t}=\left[1-\varepsilon_{i}(t)\right] V_{i+1}^{R}(t)-\left[1-\varepsilon_{i-1}(t)\right] V_{i}^{R}(t) .
$$

Combining (31) with (33), the MDR error model of the twocolor system can be obtained as follows:

$$
\begin{aligned}
\frac{d e_{i}(t)}{d t}= & V_{i+1}^{R}(t)-V_{i}^{R}\left(t-t_{T i}\right) \\
& +\varepsilon_{i-1}\left(t-t_{T i}\right) V_{i}^{R}\left(t-t_{T i}\right)-\varepsilon_{i}(t) V_{i}^{R}(t), \\
L_{u i} \frac{d \varepsilon_{i}(t)}{d t}= & {\left[1-\varepsilon_{i}(t)\right] V_{i+1}^{R}(t)-\left[1-\varepsilon_{i-1}(t)\right] V_{i}^{R}(t) . }
\end{aligned}
$$

Considering $V_{i}^{R}(t)=R_{i} \omega_{i}(t)$ and $\varepsilon_{i}(t)=T_{i}(t) / A E$, we can obtain

$$
\begin{aligned}
A E \frac{d e_{i}(t)}{d t}= & A E\left(R_{i+1} \omega_{i+1}(t)-R_{i} \omega_{i}\left(t-t_{T i}\right)\right) \\
& +R_{i}\left(T_{i-1}\left(t-t_{T i}\right) \omega_{i}\left(t-t_{T i}\right)-T_{i}(t) \omega_{i}(t)\right), \\
L_{u i} \frac{d T_{i}(t)}{d t}= & A E\left(R_{i+1} \omega_{i+1}(t)-R_{i} \omega_{i}(t)\right) \\
& +T_{i-1}(t) R_{i} \omega_{i}(t)-T_{i}(t) R_{i+1} \omega_{i+1}(t) .
\end{aligned}
$$

In the practical printing process of gravure printing machines, the change of the web cross-section in different web spans is very small and negligible, so we substitute average $A$ for $A_{i}(t)$ in (35). Equation (35) shows that the MDR mathematical model of the two-color gravure printing machines is expressed by two equations representing the dynamics of tension fluctuation between units and the dynamics of the register error fluctuation, respectively. 


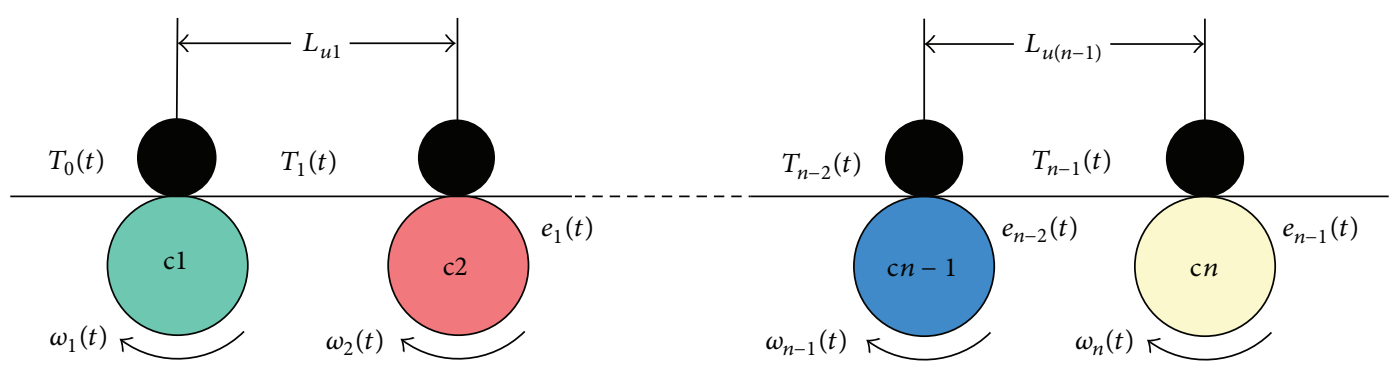

FIGURE 3: Schematic diagram of the $n$-color gravure printing machine.

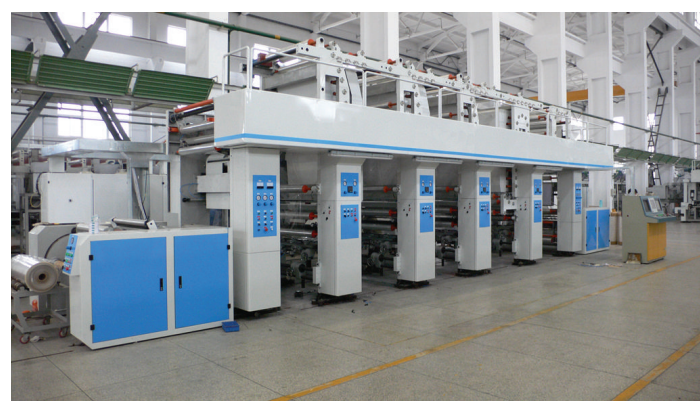

FIGURE 4: Experimental setup of the four-color gravure printing machines.

3.3. MDR System Model of the n-Color Gravure Printing Machines. The schematic diagram of the $n$-color MDR system is presented in Figure 3. $T_{0}(t)$ is the tension of the infeeding zone at steady state and can be measured by load cells.

According to (35) and Figure 3, the MDR system model of the $n$-color gravure printing machines can be expressed as follows:

$$
\begin{aligned}
A E \frac{d e_{1}(t)}{d t}= & A E\left(R_{2} \omega_{2}(t)-R_{1} \omega_{1}\left(t-t_{T 1}\right)\right) \\
& +R_{1}\left(T_{0}\left(t-t_{T 1}\right) \omega_{1}\left(t-t_{T 1}\right)-T_{1}(t) \omega_{1}(t)\right) \\
L_{u 1} \frac{d T_{1}(t)}{d t}= & A E\left(R_{2} \omega_{2}(t)-R_{1} \omega_{1}(t)\right) \\
& +T_{0}(t) R_{1} \omega_{1}(t)-T_{1}(t) R_{2} \omega_{2}(t) \\
\vdots & \\
A E \frac{d e_{n-1}(t)}{d t}= & A E\left(R_{n} \omega_{n}(t)-R_{n-1} \omega_{n-1}\left(t-t_{T(n-1)}\right)\right) \\
& +R_{n-1}\left(T_{n-2}\left(t-t_{T(n-1)}\right) \omega_{n-1}\left(t-t_{T(n-1)}\right)\right. \\
\left.-T_{n-1}(t) \omega_{n-1}(t)\right) & \\
L_{u(n-1)} \frac{d T_{n-1}(t)}{d t}= & A E\left(R_{n} \omega_{n}(t)-R_{n-1} \omega_{n-1}(t)\right) \\
& +T_{n-2}(t) R_{n-1} \omega_{n-1}(t)-T_{n-1}(t) R_{n} \omega_{n}(t)
\end{aligned}
$$

The $\omega_{i}(t)$ is composed of a stationary component $\omega^{*}$ which corresponds to web feed speed and a variable component $\Delta \omega_{i}(t)$ determined by the MDR control input. In particular, $\omega_{1}(t)$ is used as a speed reference, so we can get (37) as follows:

$$
\begin{gathered}
\omega_{1}(t)=\omega^{*}, \\
\omega_{2}(t)=\omega^{*}+\Delta \omega_{2}(t), \\
\vdots \\
\omega_{n}(t)=\omega^{*}+\Delta \omega_{n}(t) .
\end{gathered}
$$

In (36), $t_{T i}$ is also known as time delay and is defined as follows:

$$
t_{T i}=\frac{L_{u i}}{R_{i} \omega_{i}} \approx \frac{L_{u i}}{R_{i} \omega^{*}} .
$$

Equations (36) and (37) show that the $n$-color MDR system in the gravure printing machine is a multi-input multioutput, nonlinear, strong-coupling, and time-delay system. In the system, $\omega_{2}(t) \sim \omega_{n}(t)$ are the input signals, and $e_{1}(t) \sim e_{n-1}(t)$ are the output signals.

\section{Simulation and Experiments}

4.1. Experimental Setup and MDR Simulation Model of the Four-Color System. To investigate the reliability of the derived MDR mathematical model, numerical simulation and experiments under open-loop control are carried out by using MATLAB and experimental setup for the fourcolor gravure printing machines. Figures 4 and 5 show the setup configuration of the shaft-less drive gravure printing machine.

The system includes an unwinding unit, an infeeding unit, an outfeeding unit, a rewinding unit, and a four-color printing subsystem, and all of the driving shafts are driven by independent servo motors. The printing rollers are driven by servo motors (YASKAWA SGMGH-44), and printing speed is obtained by the encoder on the servo motor. The register controller (KESAI ST3000) works in shaft-less drive mode, and the MDR errors are measured using photoelectric sensors (XINMAO XM08). Tension signals are tested by load cells (MITSUBISHI LX-030TD) and tension measuring amplifiers (MITSUBISHI LM-10TA) mounted on the shaft of idle rollers. The shaft-less drive system is controlled by multiaxis 


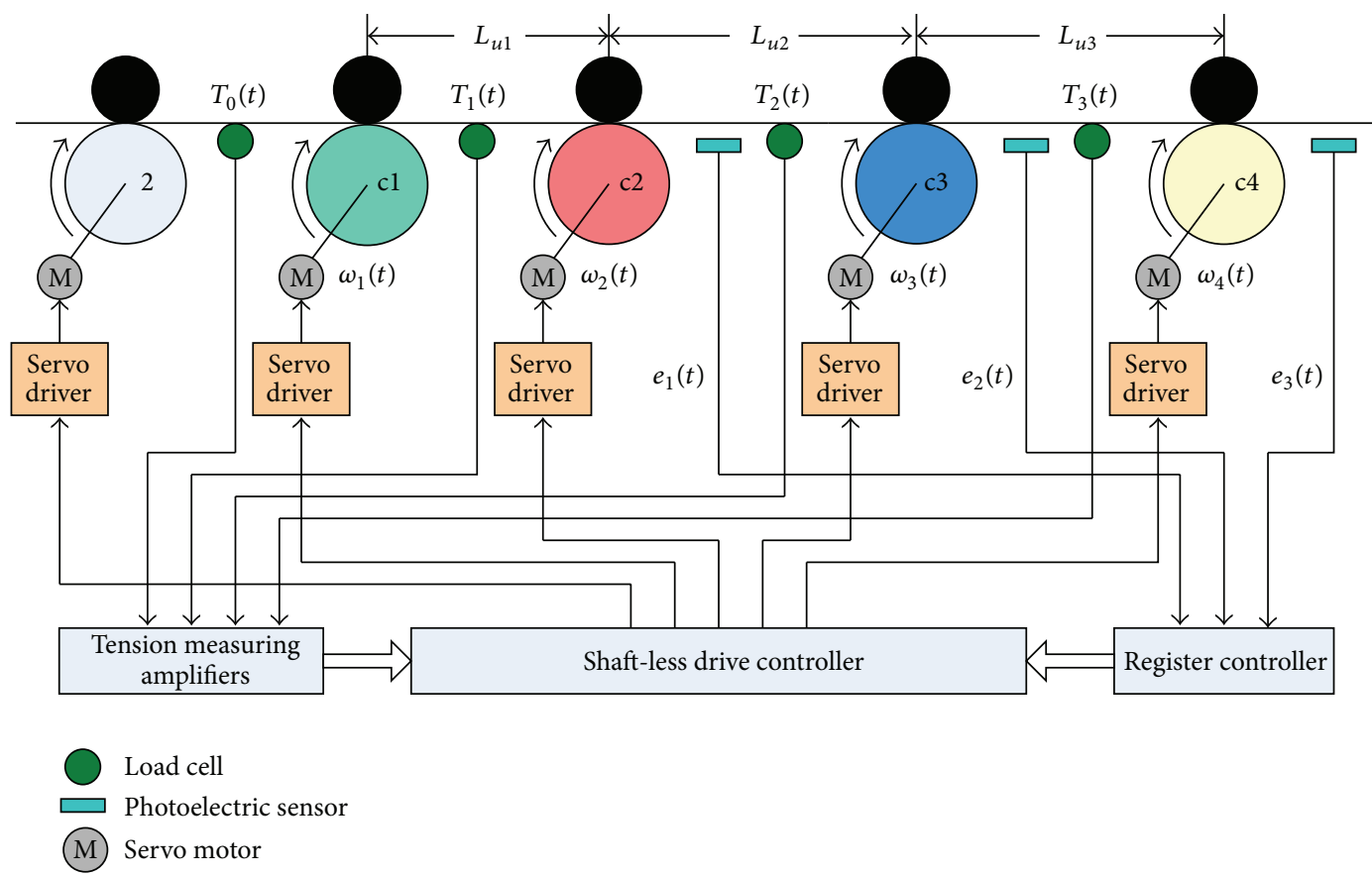

FIgURE 5: Control configuration of the experimental setup.

TABLE 1: System parameters.

\begin{tabular}{lcc}
\hline Parameters & Values & Units \\
\hline Entry tension to 1th unit $\left(T_{0}\right)$ & 100 & $\mathrm{~N}$ \\
Web length $\left(L_{u i}\right)$ & 9.1 & $\mathrm{~m}$ \\
Radius of the printing roller $\left(R_{i}\right)$ & 0.2 & $\mathrm{~m}$ \\
Web cross-section area $(A)$ & $2.0 \times 10^{-5}$ & $\mathrm{~m}^{2}$ \\
Modulus of elasticity of the web $(E)$ & 160 & $\mathrm{MPa}$ \\
\hline
\end{tabular}

controller (GOOGOLTECH T8VME). The experimental web material is biaxially oriented polypropylene (BOPP). As shown in Figure 5, tension of the printing subsystem is same with that in the infeeding zone at steady state and is not controlled; in other words, $T_{1}(t), T_{2}(t)$, and $T_{3}(t)$ are not controlled. In the infeeding unit, $T_{0}(t)$ is adjusted by changing the speed of the roller 2 . After preregistration, $e_{1}(t), e_{2}(t)$, and $e_{3}(t)$ work in the open-loop control mode and are sampled once per revolution of the printing rollers. The parameters of the experimental setup are summarized in Table 1.

According to Table 1, we can get

$$
\begin{gathered}
R_{1}=R_{2}=R_{3}=R_{4}=R, \\
L_{u 1}=L_{u 2}=L_{u 3}=L_{u}, \\
t_{T 1}=t_{T 2}=t_{T 3}=t_{T} .
\end{gathered}
$$

Hence, combining (36) with (39), the MDR system model of the four-color gravure printing machines in the simulation can be expressed as follows:

$$
\begin{aligned}
A E \frac{d e_{1}(t)}{d t}= & \operatorname{AER}\left(\omega_{2}(t)-\omega_{1}\left(t-t_{T}\right)\right) \\
& +R\left(T_{0}\left(t-t_{T}\right) \omega_{1}\left(t-t_{T}\right)-T_{1}(t) \omega_{1}(t)\right)
\end{aligned}
$$

$$
\begin{aligned}
A E \frac{d e_{2}(t)}{d t}= & A E R\left(\omega_{3}(t)-\omega_{2}\left(t-t_{T}\right)\right) \\
& +R\left(T_{1}\left(t-t_{T}\right) \omega_{2}\left(t-t_{T}\right)-T_{2}(t) \omega_{2}(t)\right), \\
A E \frac{d e_{3}(t)}{d t}= & A E R\left(\omega_{4}(t)-\omega_{3}\left(t-t_{T}\right)\right) \\
& +R\left(T_{2}\left(t-t_{T}\right) \omega_{3}\left(t-t_{T}\right)-T_{3}(t) \omega_{3}(t)\right), \\
L_{u} \frac{d T_{1}(t)}{d t}= & A E R\left(\omega_{2}(t)-\omega_{1}(t)\right) \\
& +R\left(T_{0}(t) \omega_{1}(t)-T_{1}(t) \omega_{2}(t)\right), \\
L_{u} \frac{d T_{2}(t)}{d t}= & A E R\left(\omega_{3}(t)-\omega_{2}(t)\right) \\
& +R\left(T_{1}(t) \omega_{2}(t)-T_{2}(t) \omega_{3}(t)\right), \\
L_{u} \frac{d T_{3}(t)}{d t}= & A E R\left(\omega_{4}(t)-\omega_{3}(t)\right) \\
& +R\left(T_{2}(t) \omega_{3}(t)-T_{3}(t) \omega_{4}(t)\right) .
\end{aligned}
$$

4.2. Results and Analysis of the Simulation and Experiments. In order to ensure the consistency of the simulation and experiments, the parameters used in the numerical simulation are taken from Table 1. Because the simulation and experiments under open-loop control are carried out, the speed of all servo motors is the same in the four-color printing system. In order to verify the developed MDR system model of the $n$-color gravure printing machines, $T_{0}(t)$ has a step change from $100 \mathrm{~N}$ to $110 \mathrm{~N}$ at $t=5 \mathrm{~s}$ when $\omega_{1}$ is equal to $100 \mathrm{r} / \mathrm{min}$ and $200 \mathrm{r} / \mathrm{min}$. The responses of the MDR errors 


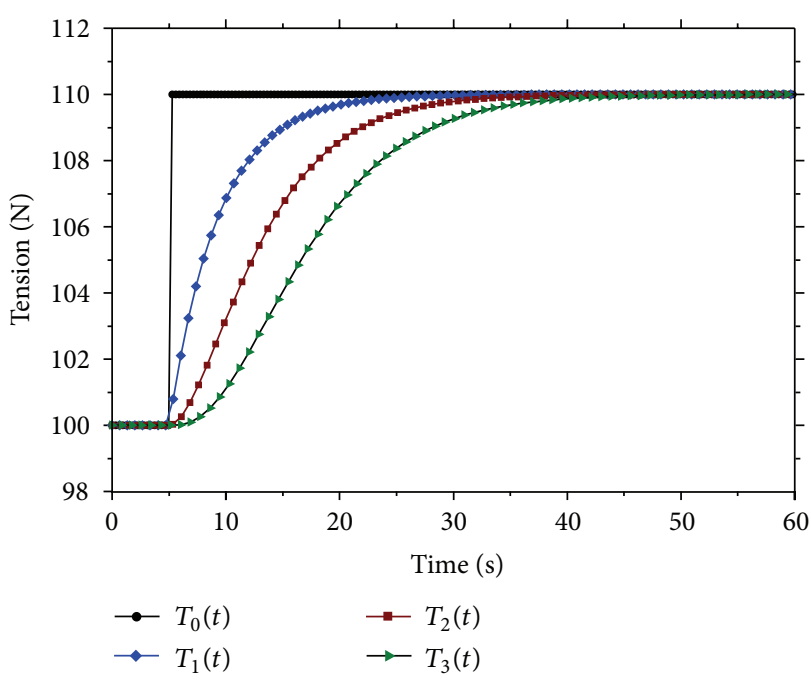

(a) Tension dynamics at $\omega_{1}=100 \mathrm{r} / \mathrm{min}$

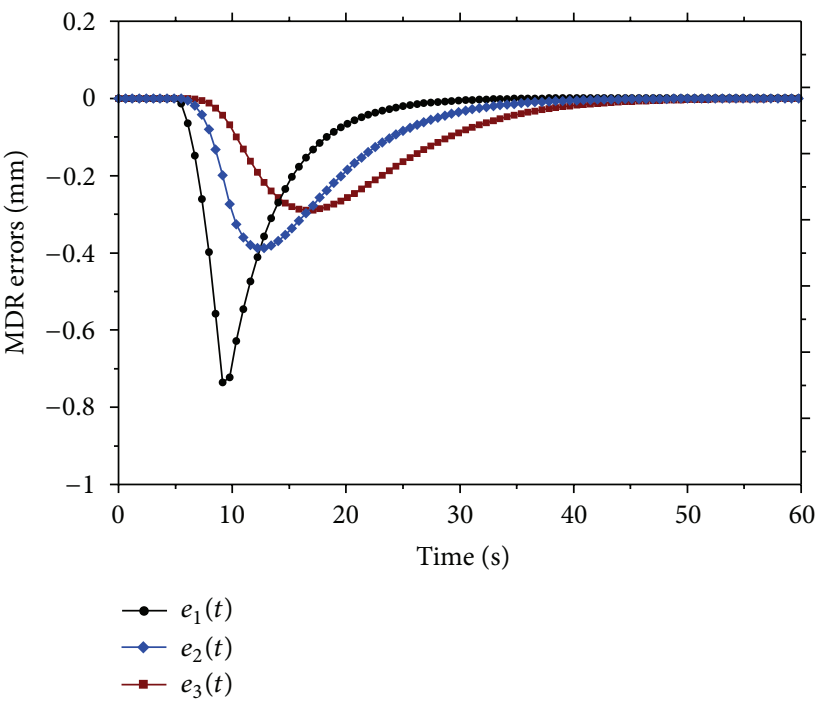

(c) MDR errors dynamics at $\omega_{1}=100 \mathrm{r} / \mathrm{min}$

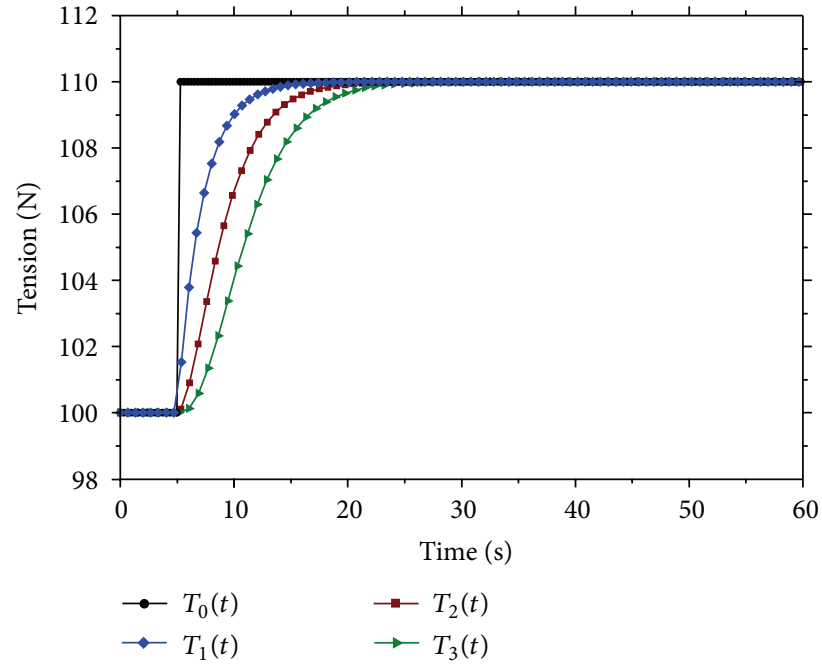

(b) Tension dynamics at $\omega_{1}=200 \mathrm{r} / \mathrm{min}$

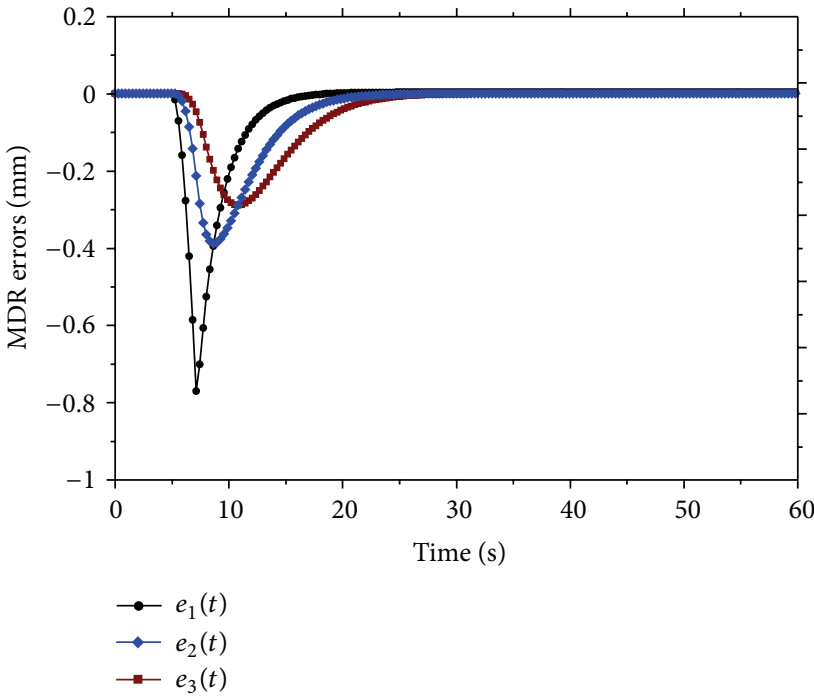

(d) MDR errors dynamics at $\omega_{1}=200 \mathrm{r} / \mathrm{min}$

Figure 6: Open-loop dynamics of the MDR errors in simulation.

and tension signals in simulation and experiments are shown in Figures 6 and 7, respectively.

As shown in Figure 6, the step change of $T_{0}(t)$ causes the step change of $T_{1}(t), T_{2}(t)$, and $T_{3}(t)$, and with an increase in $\omega_{1}(t)$, the time that $T_{1}(t), T_{2}(t)$, and $T_{3}(t)$ take to reach the steady state value is gradually shortened. For example, when $\omega_{1}(t)$ is equal to $100 \mathrm{r} / \mathrm{min}$ and $200 \mathrm{r} / \mathrm{min}, T_{1}(t)$ reaches $110 \mathrm{~N}$ at $35 \mathrm{~s}$ and $20 \mathrm{~s}$, respectively. The step change of $T_{0}(t)$ also directly causes the change of $e_{1}(t)$ and then the changes of $e_{2}(t)$ and $e_{3}(t)$ by coupling characteristics, and as the speed increases, the interval from the peak of $e_{i}(t)$ to the peak of $e_{i+1}$ $(t)$ is gradually shortened; for example, when $\omega_{1}(t)$ is equal to $100 \mathrm{r} / \mathrm{min}$ and $200 \mathrm{r} / \mathrm{min}$, the interval from the peak of $e_{1}(t)$ to the peak of $e_{2}(t)$ is $3.11 \mathrm{~s}$ and $1.56 \mathrm{~s}$, respectively.

Figures 6 and 7 illustrate that although there are some disturbances in the experiments, the experimental results are almost coincident with the simulation curves. For example, with an increase in $\omega_{1}(t)$, the time that tension signals take to reach the steady state value is gradually shortened and so on. Comparing with Figures 6(c), 6(d), 7(c), and 7(d), respectively, the peak of $e_{i}(t)$ in the experiments is slightly more than that in the simulation, such as when $\omega_{1}(t)$ is equal to $200 \mathrm{r} / \mathrm{min}$, the peaks of $e_{1}(t)$ in the experiments and simulation are $-0.82 \mathrm{~mm}$ and $-0.78 \mathrm{~mm}$, respectively. There are two primary reasons: one is that there are external disturbances from environment in the experiments, and the other is that there are errors between the simulation parameters and real experimental parameters.

The results of the simulation and experiments indicate that the established MDR system model is accurate and reliable and can be used to design a MDR controller. At the same time, the results also offer a powerful proof that the 


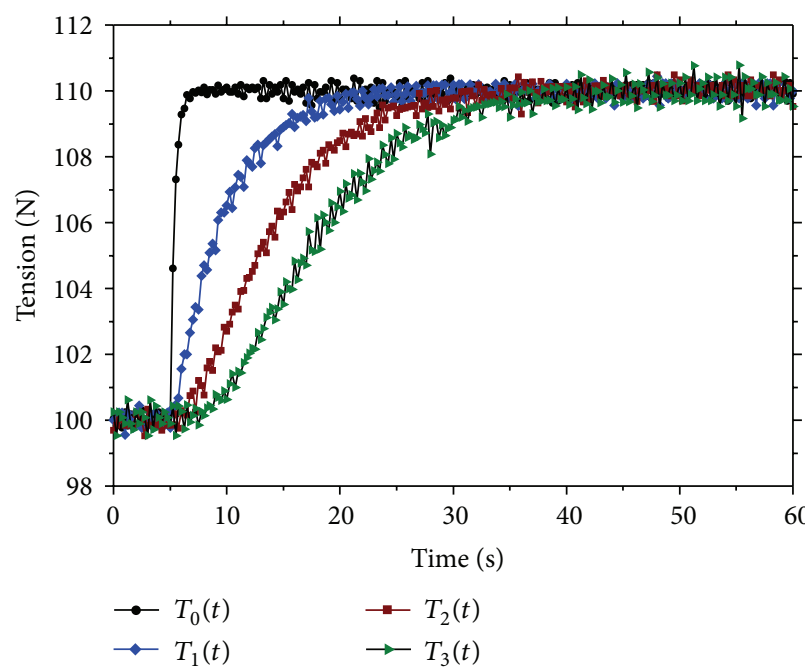

(a) Tension dynamics at $\omega_{1}=100 \mathrm{r} / \mathrm{min}$

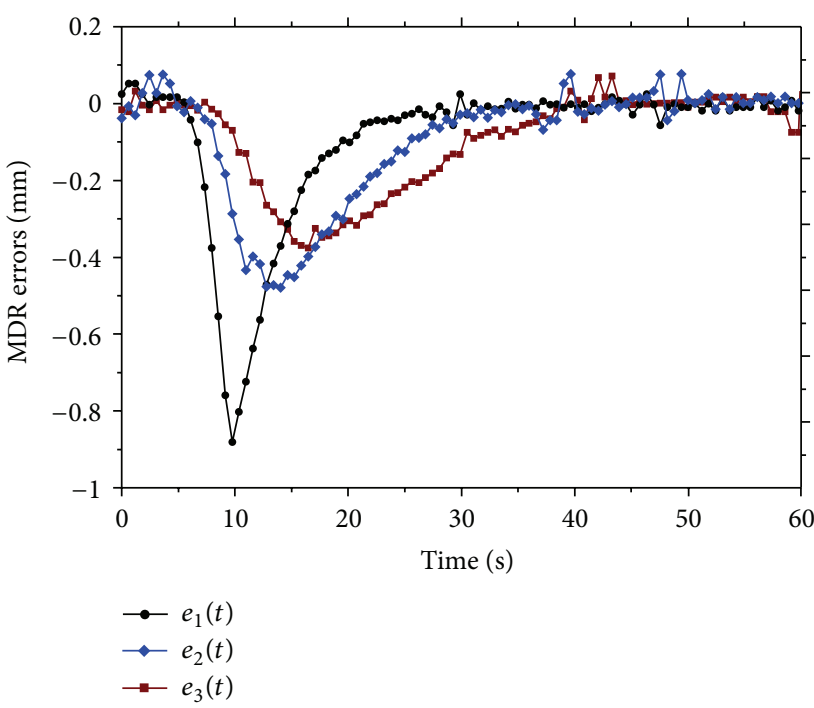

(c) MDR errors dynamics at $\omega_{1}=100 \mathrm{r} / \mathrm{min}$

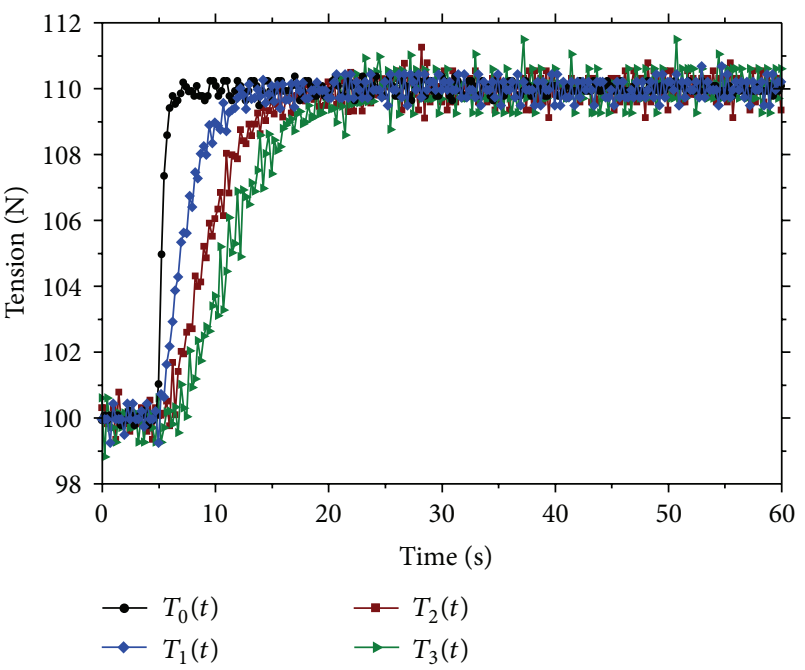

(b) Tension dynamics at $\omega_{1}=200 \mathrm{r} / \mathrm{min}$

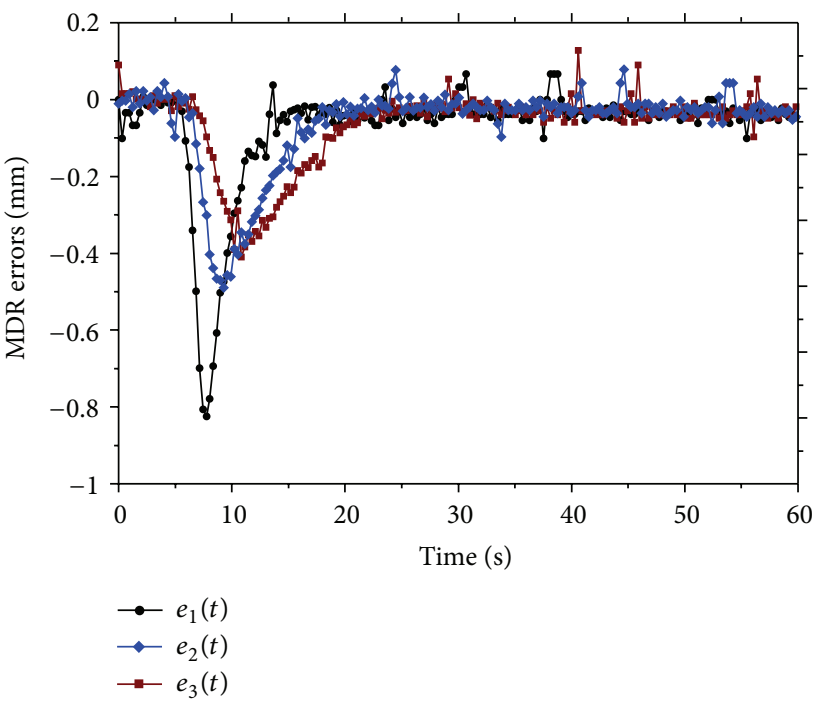

(d) MDR errors dynamics at $\omega_{1}=200 \mathrm{r} / \mathrm{min}$

FiguRE 7: Open-loop dynamics of the MDR errors in experiments.

MDR system of the multicolor gravure printing machines is a multi-input multioutput, nonlinear, strong-coupling, and time-delay system.

\section{Conclusions}

In the gravure printing machines, the register accuracy is the most important in performance. Therefore, in order to improve the control accuracy of MDR system, a nonlinear mathematical model of the MDR system in shaft-less drive mode is presented and derived in detail for the multicolor gravure printing machines in this paper. First, we introduce the schematic diagram of the shaft-less drive gravure printing machines. Secondly, we make a deep analysis of the causes and influencing factors of the MDR errors, then establish the MDR error of the two-color system, and further obtain the MDR system model of the $n$-color gravure printing machines.
At last, the proposed model is verified by the numerical simulations and experimental studies. Therefore, it can be concluded that the MDR system model is accurate enough to describe dynamics of the MDR errors in multicolor printing system and is worthwhile to be developed as an effective MDR controller in future study.

\section{Nomenclature}

$\alpha_{i}$ : Phase angle of the color mark on the roller ci

$t_{i}$ : Printing moment of the color mark on the roller ci

$t_{T i}$ : Transmission time of the color mark from $i$ th to $(i+1)$ th printing units

$\omega_{i}$ : Angular velocity of rotation of the roller $c i$ 
$X_{F i}$ : Coordinate on $x$-axis of the color mark on the roller $\mathrm{c} i$

$X_{B i}$ : Coordinate on $x$-axis of the color mark on the web

$e_{i}$ : $\quad$ Register error between $i$ th and $(i+1)$ th printing units

$R_{i}$ : The radius of the roller $\mathrm{c} i$

$L_{u i}$ : Length of the free web between $i$ th and $(i+1)$ th printing units

$L_{i}$ : Actual length of the web between $i$ th and $(i+1)$ th printing units

$\Delta L_{i}$ : The length variation of the web between $i$ th and $(i+1)$ th printing units

$\varepsilon_{i}$ : $\quad$ Strain of the web between $i$ th and $(i+1)$ th printing units

$T_{i}$ : Tension of the web between $i$ th and $(i+1)$ th printing units

$V_{i}^{T}$ : Virtual velocity of the roller $\mathrm{ci}$

$V_{i}^{R}$ : Web velocity of the roller $c i$

$E$ : The modulus of elasticity of the web material

$\rho_{i}$ : $\quad$ Density of the web between $i$ th and $(i+1)$ th printing units

$A_{i}$ : Cross-section of the web between $i$ th and $(i+1)$ th printing units.

\section{Conflict of Interests}

The authors declare that they have no financial and personal relationships with other people or organizations that can inappropriately influence our work; there is no professional or other personal interest of any nature or kind in any product or company that could be construed as influencing the position presented in, or the review of, the paper.

\section{Acknowledgments}

This research is supported by the Program for Changjiang Scholars (IRT1172) and the National Science \& Technology Pillar Program of China, under Grant no. 2012BAF13B06.

\section{References}

[1] G. Brandenburg, "New mathematical models for web tension and register error," Noordnederlands Boekbedrijb, vol. 1, pp. 411438, 1977.

[2] T. Yoshida, S. Takagi, T. Shen, and Y. Muto, "Modeling and cooperative resister control of gravure printing press," Nihon Kikai Gakkai Ronbunshu C, vol. 74, no. 2, pp. 339-345, 2008.

[3] T. Yoshida, S. Takagi, Y. Muto, and T. Shen, "Register control of rotogravure printing press-Application of nonlinear control theory to sectional drive printing press," IEEJ Transactions on Industry Applications, vol. 128, no. 9, pp. 1133-1139, 2008.

[4] T. Yoshida, S. Takagi, Y. Muto, and T. Shen, "Register control of rotogravure printing press. Application of nonlinear control theory to sectional drive printing press," Electronics and Communications in Japan, vol. 94, no. 1, pp. 17-24, 2011.

[5] D. Z. Hua, L. Xi, and Y. Xiaomeng, "Model and control for gravure register printing system," in Proceedings of the 26th
Chinese Control Conference (CCC '07), pp. 277-280, Zhangjiajie, China, July 2007.

[6] J. Li, X. Mei, T. Tao, and S. Liu, "Research on the register system modelling and control of gravure printing press," Proceedings of the Institution of Mechanical Engineers C, vol. 226, no. 3, pp. 626-635, 2012.

[7] H. Kang, C. Lee, and K. Shin, "A novel cross directional register modeling and feedforward control in multi-layer roll-to-roll printing," Journal of Process Control, vol. 20, no. 5, pp. 643-652, 2010.

[8] H.-K. Kang, C.-W. Lee, J.-M. Lee, and K.-H. Shin, "Cross direction register modeling and control in a multi-layer gravure printing," Journal of Mechanical Science and Technology, vol. 24, no. 1, pp. 391-397, 2010.

[9] H. Wang, D. Logghe, and D. Miskin, "Physical modelling and control of lateral web position for wallpaper making processes," Control Engineering Practice, vol. 13, no. 4, pp. 401-412, 2005.

[10] H.-K. Kang, C.-W. Lee, and K.-H. Shin, "Novel modeling of correlation between two-dimensional registers in large-area multilayered roll-to-roll printed electronics," Japanese Journal of Applied Physics, vol. 50, no. 1, Article ID 016701, 7 pages, 2011.

[11] H. Kang, C. Lee, J. Lee, and K. Shin, “Two-dimensional register modeling and control in multi-layer roll-to-roll e-printing systems," in Proceedings of the 18th IFAC World Congress, vol. 18, pp. 6763-6770, 2011.

[12] S. Liu, X. Mei, F. Kong, and J. Shen, “Tension controller design for unwinding tension system based on active disturbance rejection control," in Proceedings of the IEEE International Conference on Mechatronics and Automation,, pp. 1798-1803, Chengdu, China, 2012. 


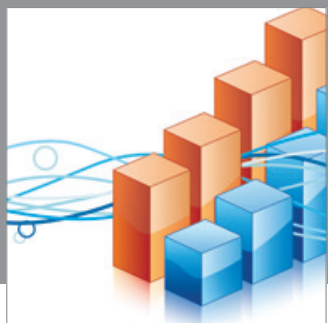

Advances in

Operations Research

mansans

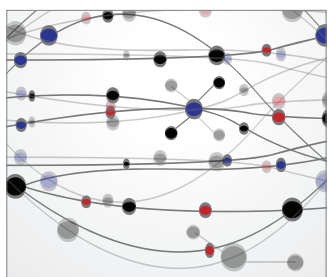

The Scientific World Journal
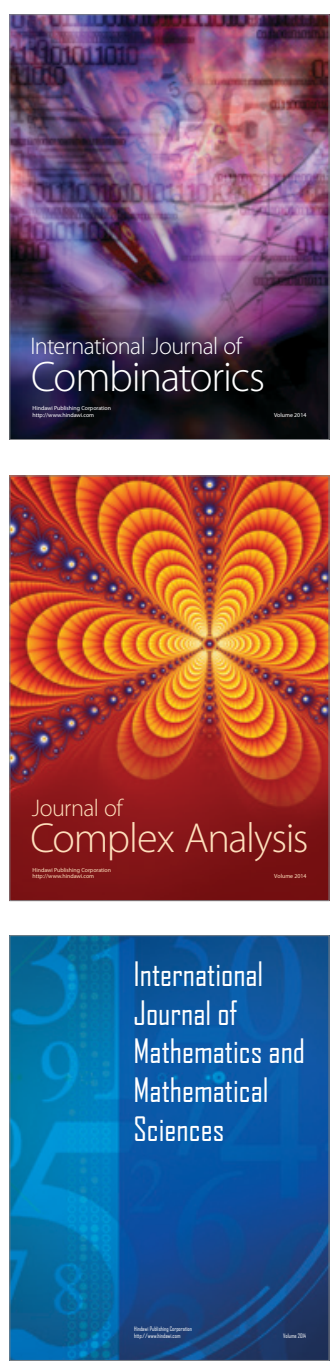
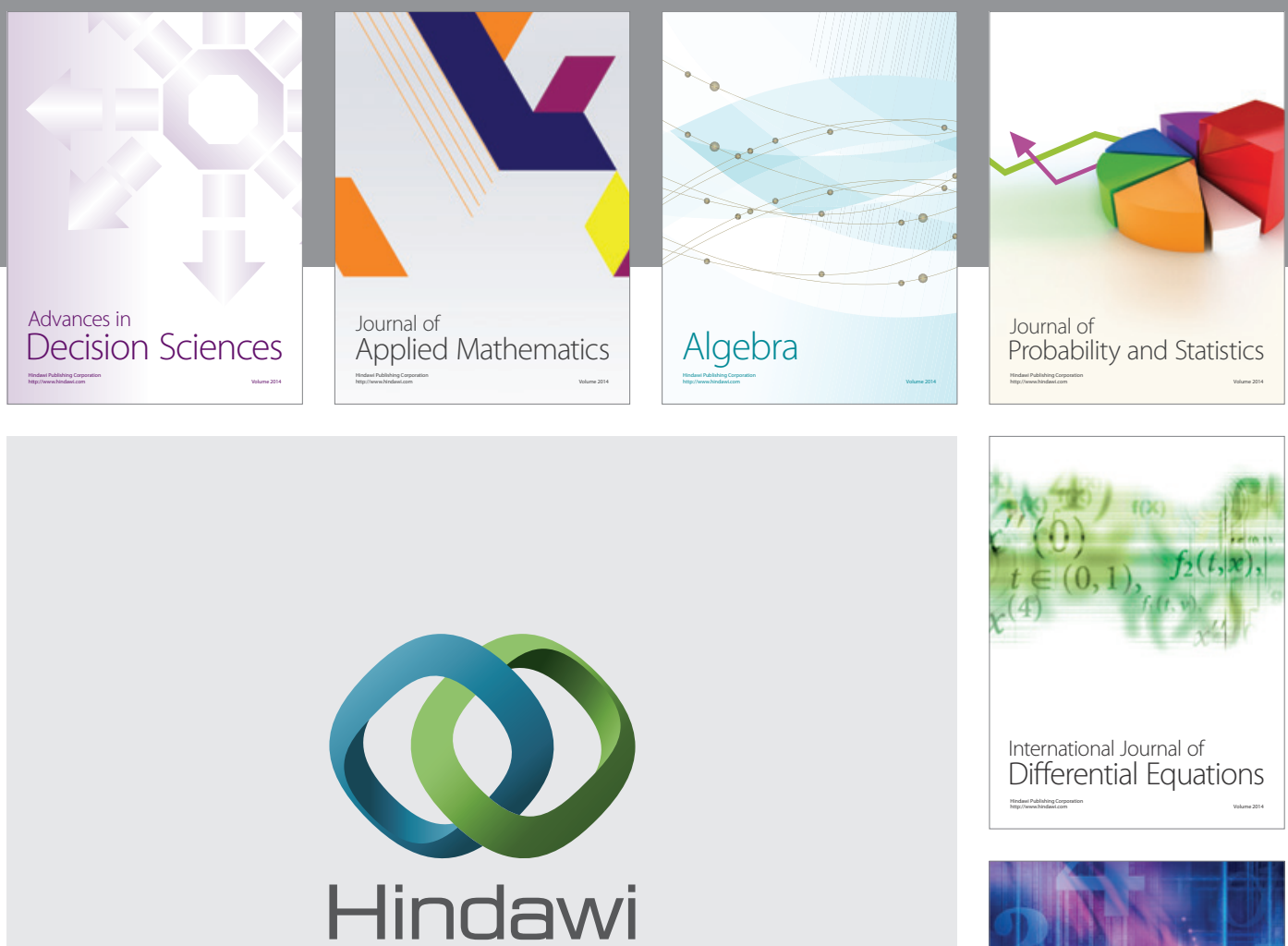

Submit your manuscripts at http://www.hindawi.com
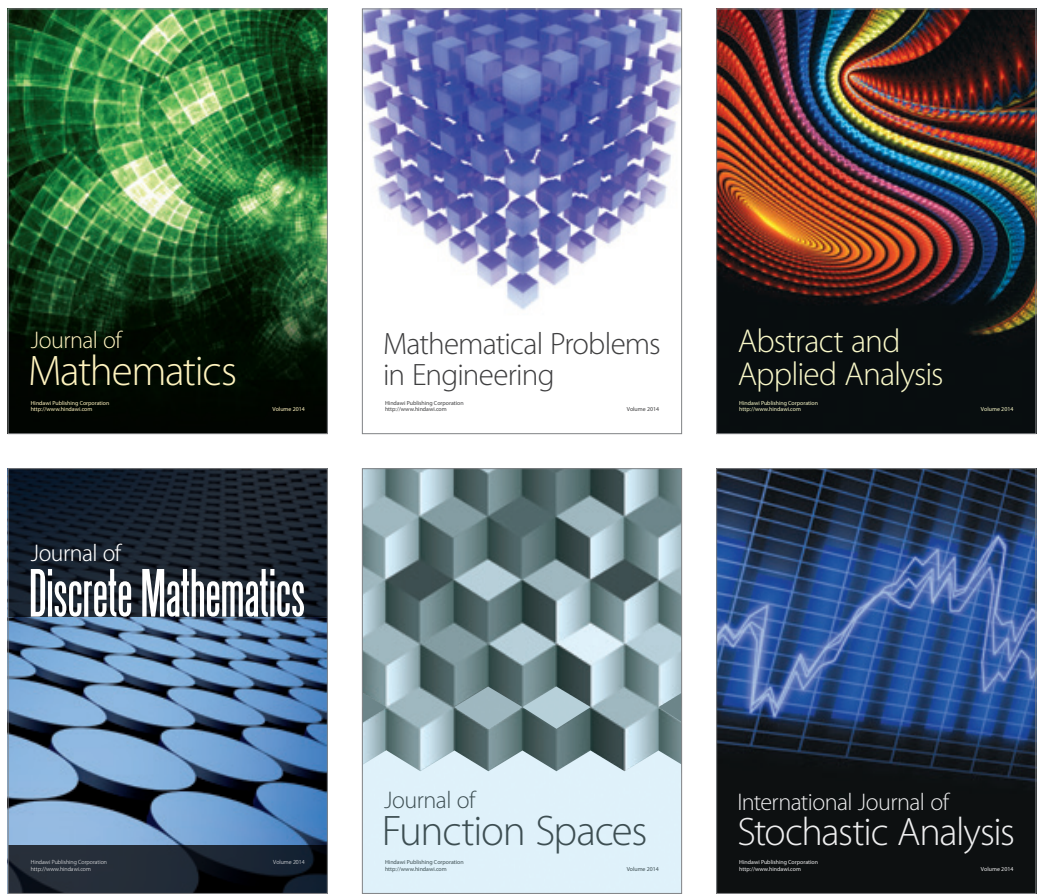

Journal of

Function Spaces

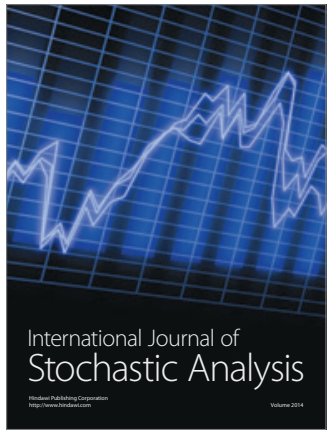

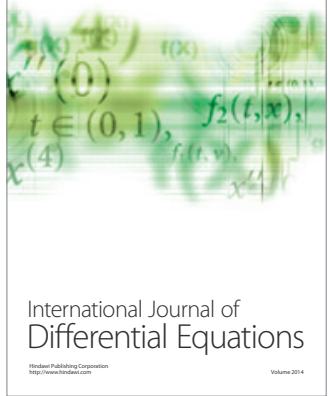
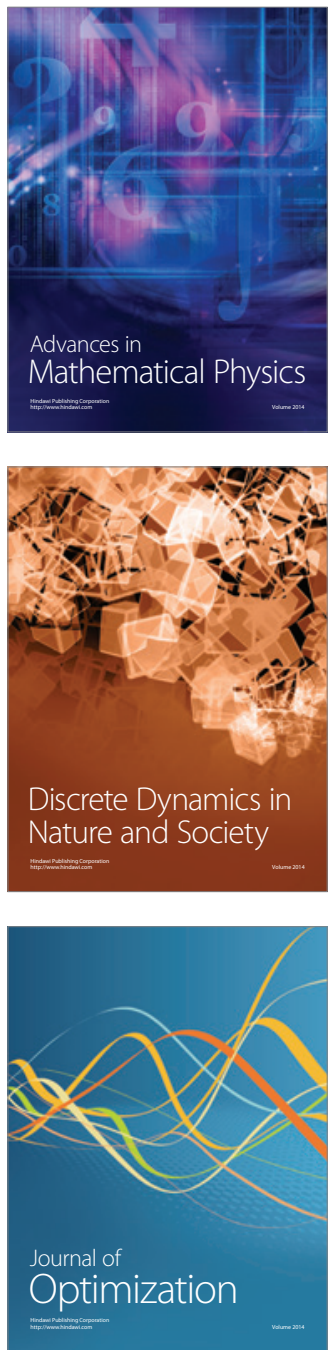